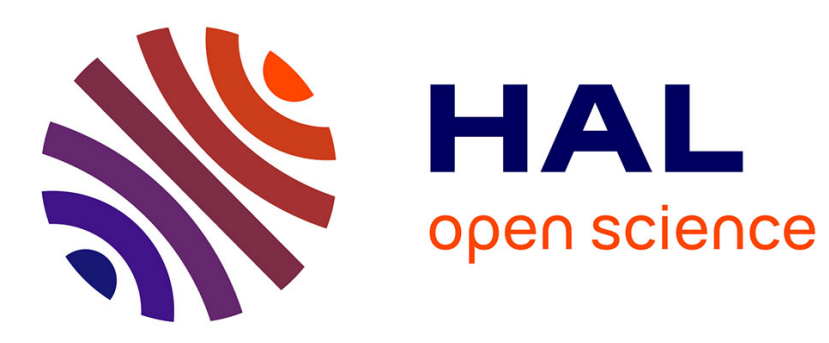

\title{
Identification of switched linear state space models without minimum dwell time
}

Laurent Bako, Guillaume Mercère, René Vidal, Stéphane Lecoeuche

\section{To cite this version:}

Laurent Bako, Guillaume Mercère, René Vidal, Stéphane Lecoeuche. Identification of switched linear state space models without minimum dwell time. IFAC Symposium on System Identification, Jul 2009, Saint Malo, France. pp.SYSID09. hal-00374098

\section{HAL Id: hal-00374098 https://hal.science/hal-00374098}

Submitted on 7 Apr 2009

HAL is a multi-disciplinary open access archive for the deposit and dissemination of scientific research documents, whether they are published or not. The documents may come from teaching and research institutions in France or abroad, or from public or private research centers.
L'archive ouverte pluridisciplinaire HAL, est destinée au dépôt et à la diffusion de documents scientifiques de niveau recherche, publiés ou non, émanant des établissements d'enseignement et de recherche français ou étrangers, des laboratoires publics ou privés. 


\title{
Identification of switched linear state space models without minimum dwell time
}

\author{
L. Bako* G. Mercère ${ }^{* *}$ R. Vidal ${ }^{* * *}$ and S. Lecœuche* \\ * Ecole des Mines de Douai, Département Informatique et Automatique, \\ 59508 Douai, France \\ ** Université de Poitiers, Laboratoire d'Automatique et d'Informatique \\ Industrielle, 86022 Poitiers, France \\ *** Center for Imaging Science, The Johns Hopkins University, Baltimore, \\ MD 21218, USA
}

\begin{abstract}
We consider the problem of identifying switched linear state space models from a finite set of input-output data. This is a challenging problem, which requires inferring both the discrete state and the parameter matrices associated with each discrete state. An important contribution of our work is that we do not make the restrictive assumption of minimum dwell time between the switches, as it is customary in methods that deal with such models. We first propose a technique for eliminating the unknown continuous state from the model equations under an appropriate assumption of observability. On a time horizon, this gives us a new switched input-output relation that involves structured intermediary matrices, which depend on the state space representation matrices. To estimate the intermediary matrices, we present a randomly initialized algorithm that alternates between data classification and parameter update via recursive least squares. Given these matrices, the parameters associated to the different discrete states can be computed after a correct estimation of the discrete state.
\end{abstract}

\section{INTRODUCTION}

Hybrid system identification refers to the problem of identifying a set of interacting dynamical submodels from input-output data. Due to the numerous potential applications of hybrid models, many works have recently addressed this problem. Most of them are concerned with the estimation of input-output models such as PieceWise Auto-Regressive eXogenous (PWARX) models [1-4] or Switched ARX models [5-7]. A comprehensive survey on the developed techniques can be found in [8].

However, in some situations it may be desirable to obtain state space models directly from data. For example, notions such as observability and controllability are well established and usually studied based on state space models. These latter models are often also more convenient for describing Multiple Input-Multiple Output (MIMO) systems as they provide a nice and compact representation. Furthermore, there already exists a strong theory for process control, observer design, system realization and stability analysis that relies on state space models.

The main difficulty in solving the problem of identifying switched state space models lies in the fact that the discrete state, the continuous state and the parameters of the different submodels are all unknown and highly coupled. Moreover, in comparison with the estimation of switched input-output models, the identification of switched state space models suffers from the additional difficulty that the continuous state is generally unknown. Therefore, since the regressor is not completely available, direct partitioning of the input-state space in PieceWise Affine (PWA) models, for example, becomes a very hard task. Another issue in dealing with state space models is that different submodels may be identified with respect to different bases of the state space, hence one needs to make the identified submodels compatible with respect to a common basis.
Existing identification approaches generally operate under the restrictive assumption that consecutive switching times are separated by a certain minimum time that is referred to as the dwell time. Hence, when the dwell time is long enough, classic subspace identification methods can be applied between two consecutive switches [9], [10], [11]. The method reported in [12] can be applied with a relatively small dwell time, but requires the whole discrete state sequence to be completely available. This may be a very strong requirement in practice.

In this paper, we consider the identification of a switched linear system (SLS) without making the customary assumption of a minimum dwell time. Under an appropriate assumption of observability, we first convert the state space model into an inputoutput relation that theoretically involves an increased number of linear submodels. We then present a simple identification scheme that alternates between data classification and model parameter update for identifying this intermediate model. A third step of our method consists in obtaining a state space realization of the data-generating switched model. This is done by exploiting the particular structures of the previously identified intermediary matrices.

The outline of the paper is as follows. In $\S 2$ we formulate the switched linear state space model identification problem and briefly discuss the closely related issue of identifiability. A particular observability condition is introduced and illustrated through a few examples. In $\S 3$ we propose a simple method for estimating the submodels of the SLS model. In $\S 4$ we illustrate our method on a numerical example. $\S 5$ concludes the paper.

\section{PROBLEM STATEMENT}

We consider a Switched Linear System (SLS) described by the following state space model 


$$
\left\{\begin{aligned}
x(t+1) & =A_{\lambda_{t}} x(t)+B_{\lambda_{t}} u(t) \\
y(t) & =C_{\lambda_{t}} x(t)+D_{\lambda_{t}} u(t)
\end{aligned}\right.
$$

where $\lambda_{t} \in \mathcal{S}=\{1, \ldots, s\}$ refers to the discrete state of the system, $s$ is the number of submodels, $n$ stands for the system order (dimension of the state space) that is assumed to be the same for all the submodels. The vectors $x(t) \in \mathbb{R}^{n}$, $u(t) \in \mathbb{R}^{n_{u}}$, and $y(t) \in \mathbb{R}^{n_{y}}$ are respectively the continuous state, the input and the output of the system. The matrices $A_{\lambda_{t}}, B_{\lambda_{t}}, C_{\lambda_{t}}, D_{\lambda_{t}}$ are the parameter matrices associated with the submodel indexed by $\lambda_{t}$. In the method to be presented, no constraint is imposed on the switching mechanism. That is, the switches can be exogenous, deterministic, state-driven, eventdriven, time-driven or totally random. However, we assume throughout the paper that the order $n$ and the number of submodels $s$ are available a priori.

Given input-output data $\{u(t), y(t)\}_{t=1}^{N}$ generated by an SLS of the form (1), the number of submodels $s$ and the order $n$, we are interested in estimating a (any) realization $\left\{A_{j}, B_{j}, C_{j}, D_{j}\right\}_{j=1}^{s}$ of the system (1).

In order to solve this problem, it is important to first analyze whether it is well-posed. In other words, we are interested in the conditions under which it may be possible to infer a model of the form (1) from data. This problem is generally referred to as realization theory [13]. In contrast to the realization of linear systems, which is now well understood, the realization of hybrid system is still a widely open problem. Some interesting works that introduce the subject are [13-16].

\subsection{Input-output behavior of switched models}

In this subsection, we briefly illustrate some issues pertaining to the realizability of the input-output behavior associated with the switched model (1). As in the case of linear systems, there exist infinitely many switched state space models that produce the same input-output trajectory as (1). To see this, let us rewrite the output $y(t)$ in (1) in the following form

$$
\begin{aligned}
y(t) & =C_{\lambda_{t}} A_{\lambda_{t-1}} \cdots A_{\lambda_{0}} x(0)+C_{\lambda_{t}} A_{\lambda_{t-1}} \cdots A_{\lambda_{1}} B_{\lambda_{0}} u(0) \\
& +C_{\lambda_{t}} A_{\lambda_{t-1}} \cdots A_{\lambda_{2}} B_{\lambda_{1}} u(1)+\cdots \\
& +C_{\lambda_{t}} B_{\lambda_{t-1}} u(t-1)+D_{\lambda_{t}} u(t) .
\end{aligned}
$$

If in this equation we replace matrices $A_{\lambda_{t}}, B_{\lambda_{t}}$, and $C_{\lambda_{t}}$ by matrices $T_{\lambda_{t+1}} A_{\lambda_{t}} T_{\lambda_{t}}^{-1}, T_{\lambda_{t+1}} B_{\lambda_{t}}$, and $C_{\lambda_{t}} T_{\lambda_{t}}^{-1}$, respectively, where $\left\{T_{\lambda_{k}}\right\}$ is a sequence of nonsingular matrices, and if we additionally substitute $T_{\lambda_{0}} x(0)$ for $x(0)$, then the input-output map of the system remains unchanged. Note that the same remark still holds even when the matrices $\left\{T_{\lambda_{t}}\right\}$ are replaced by $\left\{T_{t}\right\}$, that is, when they are indexed by $t \in \mathbb{Z}$.

Following the work of [14], we can notice that the ambiguity of model (1) from data even lies in some further structural characteristics of the model. In fact, given a sufficiently long sequence of data generated by (1), neither the order $n$ nor the number $s$ of submodels are uniquely determined. This means that there may exist models $\left\{A_{j}, B_{j}, C_{j}, D_{j}\right\}_{j=1}^{\bar{s}}$ of order $\bar{n}$ such that $\bar{n} \leq n$ and $\bar{s} \geq s$, or $\bar{n} \geq n$ and $\bar{s} \leq s$, that re-create the data [14].

In short, there are three main factors that justify the indeterminacy of the SLS model from data: the sequence of discrete states and the number of submodels; the order or the dimension of the state space; the multiplicity of possible state coordinates bases. In the case of linear models (one single submodel), the ambiguity on the order $n$ may be removed by requiring that the identified model be minimal, i.e. observable and controllable. The remaining problem of multiple bases for the state space can be overcome by arbitrarily specifying one coordinate basis.

In the case of SLS, a notion of minimality may require one to impose upper bounds on both the number of submodels and the order in order to reduce the ambiguity. Unfortunately this notion is still under formalization in the existing literature [13]. Nevertheless, we know from linear system theory that the system order can be constrained by the concept of observability.

\subsection{Pathwise observability}

Observability of a switched model refers generally to the possibility of uniquely inferring its state $\left(\lambda_{t}, x(t)\right)$ from observed data on a certain time horizon. Depending on the considered observation horizon and on whether one wishes to observe the discrete state $\lambda_{t}$ and/or the continuous state $x(t)$, many notions of observability are defined in the literature. We refer interested readers to $[14,17]$, for example. Here, our objective is not to study extensively the problem of observability, but rather to just recall a particular notion of observability that will be useful during the identification process.

To begin with, consider an arbitrary sequence of discrete states $\bar{\lambda}_{t, f}=\lambda_{t} \cdots \lambda_{t+f-1} \in \mathcal{S}^{f}=\mathcal{S} \times \cdots \times \mathcal{S}$, where $f$ is some integer. For each sequence $\bar{\lambda}_{t, f}$, we define the observability matrix $\Gamma\left(\bar{\lambda}_{t, f}\right)$ as

$$
\Gamma\left(\bar{\lambda}_{t, f}\right)=\left[\begin{array}{c}
C_{\lambda_{t}} \\
C_{\lambda_{t+1}} A_{\lambda_{t}} \\
\vdots \\
C_{\lambda_{t+f-1}} A_{\lambda_{t+f-2}} \cdots A_{\lambda_{t}}
\end{array}\right],
$$

and the matrix $H\left(\bar{\lambda}_{t, f}\right)$ as

$$
\begin{gathered}
H\left(\bar{\lambda}_{t, f}\right)= \\
{\left[\begin{array}{cccc}
D_{\lambda_{t}} & 0 & \ldots & 0 \\
C_{\lambda_{t+1}} B_{\lambda_{t}} & D_{\lambda_{t+1}} & \ldots & 0 \\
\vdots & \vdots & \ddots & \vdots \\
C_{\lambda_{t+f-1}} A_{\lambda_{t+f-2}} \ldots A_{\lambda_{t+1}} B_{\lambda_{t}} & (*) & \ldots & D_{\lambda_{t+f-1}}
\end{array}\right],}
\end{gathered}
$$

where (*) stands for $C_{\lambda_{t+f-1}} A_{\lambda_{t+f-2}} \ldots A_{\lambda_{t+2}} B_{\lambda_{t+1}}$. If we define the vectors

$$
\begin{aligned}
& y_{f}(t)=\left[\begin{array}{lll}
y(t)^{\top} & \cdots & y(t+f-1)^{\top}
\end{array}\right]^{\top} \in \mathbb{R}^{f n_{y},}, \\
& u_{f}(t)=\left[\begin{array}{lll}
u(t)^{\top} & \cdots & u(t+f-1)^{\top}
\end{array}\right]^{\top} \in \mathbb{R}^{f n_{u}},
\end{aligned}
$$

then, for all $t \geq 0$, we can obtain from (1),

$$
y_{f}(t)=\Gamma\left(\bar{\lambda}_{t, f}\right) x(t)+H\left(\bar{\lambda}_{t, f}\right) u_{f}(t) .
$$

We would like to determine the state $x(t)$ uniquely as a function of $u_{f}(t)$ and $y_{f}(t)$ for all $t \geq 0$, where $f$ is a certain fixed integer. In view of equation (6), such a property depends exclusively on the rank of $\Gamma\left(\bar{\lambda}_{t, f}\right)$. More precisely, we state the following definition.

Definition 1. ([17]). The SLS (1) is said to be pathwise observable if there is an integer $m$ verifying $\operatorname{rank}\left(\Gamma\left(\bar{\lambda}_{t, m}\right)\right)=n$ for any discrete state sequence $\bar{\lambda}_{t, m}$ of length $m$. The smallest such number $m$ is called the observability index.

Throughout the paper, we denote the observability index with $\nu=\min \left\{m: \operatorname{rank}\left(\Gamma\left(\bar{\lambda}_{t, m}\right)\right)=n \forall t\right\}<\delta_{\nu}$ for a certain finite number $\delta_{\nu}$ that is assumed to be relatively small. 
Next, we give two examples of situations where the seemingly strong observability condition of Definition 1 holds.

Theorem 1. (Observability of MISO switched systems).

Assume that the system (1) is a MISO, that is, $n_{y}=1$. Then (1) is pathwise observable with observability index $\nu=n$ if and only if there exists a sequence of nonsingular matrices $\left\{T_{\sigma_{t}}\right\}$ in $\mathbb{R}^{n \times n}$ satisfying for all $t$,

$$
\begin{aligned}
T_{\sigma_{t+1}} A_{\lambda_{t}} T_{\sigma_{t}}^{-1} & =\left[\begin{array}{cccc}
0 & 1 & \cdots & 0 \\
\vdots & \vdots & \ddots & \vdots \\
0 & 0 & \cdots & 1 \\
-a_{\bar{\sigma}_{t}}^{0} & -a_{\bar{\sigma}_{t}}^{1} & \cdots & -a_{\bar{\sigma}_{t}}^{n-1}
\end{array}\right] \\
C_{\lambda_{t}} T_{\sigma_{t}}^{-1} & =\left[\begin{array}{llll}
1 & 0 & \cdots & 0
\end{array}\right],
\end{aligned}
$$

where $\sigma_{t}$ and $\bar{\sigma}_{t}$ take values in some finite sets.

Theorem 1 can be generalized to certain types of MIMO switched systems. To this end, let us assume that there is a set of vectors $\left\{\gamma_{j}\right\}_{j=1}^{s} \subset \mathbb{R}^{n_{y}}$ such that for all $\bar{\lambda}_{t, n}, \operatorname{rank}\left(\Psi\left(\bar{\lambda}_{t, n}\right)\right)=$ $\operatorname{rank}\left(\Gamma\left(\bar{\lambda}_{t, n}\right)\right)$, where

$$
\begin{aligned}
& \Psi\left(\bar{\lambda}_{t, n}\right)= \\
& {\left[\begin{array}{c}
\bar{c}_{\lambda_{t+1}}^{\top} A_{\lambda_{t}} \\
\vdots \\
\bar{c}_{\lambda_{t+n-1}}^{\top} A_{\lambda_{t+n-2}} \cdots A_{\lambda_{t}}
\end{array}\right]=\left[\begin{array}{lll}
\gamma_{\lambda_{t}}^{\top} & & \\
& \ddots & \\
& & \gamma_{\lambda_{t+n-1}}^{\top}
\end{array}\right] \Gamma\left(\bar{\lambda}_{t, n}\right)}
\end{aligned}
$$

and $\bar{c}_{\lambda_{t}}^{\top}=\gamma_{\lambda_{t}}^{\top} C_{\lambda_{t}}$. For a MIMO system satisfying this assumption, pathwise observability with index $\nu=n$ holds if the switched MISO $\left\{A_{j}, \bar{c}_{j}^{\top}\right\}_{j=1}^{s}$ is pathwise observable, i.e., if and only if $A_{\lambda_{t}}$ and $\bar{c}_{\lambda_{t}}^{\top}$ can take the form (7) and (8) for any $\lambda_{t}$.

In general, observability of the individual linear submodels does not imply observability of the global SLS. However, when there is a minimum dwell time of $2 n$, the following holds.

Theorem 2. Consider a MIMO switched system represented by a model of the form (1). Assume that

- each submodel is observable i.e., $\operatorname{rank}\left(\Gamma_{n}\left(A_{j}, C_{j}\right)\right)=n$ for any $j \in \mathcal{S}$, with $\Gamma_{n}\left(A_{j}, C_{j}\right)$ defined as

$$
\Gamma_{n}\left(A_{j}, C_{j}\right)=\left[\begin{array}{lll}
C_{j}^{\top} & \left(C_{j} A_{j}\right)^{\top} \cdots\left(C_{j} A_{j}^{n-1}\right)^{\top}
\end{array}\right]^{\top},
$$

- $\operatorname{rank}\left(A_{j}\right)=n \forall j \in \mathcal{S}$,

- the switching times are separated by a minimum dwell time $\tau_{\text {dwell }} \geq 2 n$.

Then, the system (1) is pathwise observable with observability index $\nu \leq 2 n$ in the sense of Definition 1 .

Remark 1. For systems where the number of outputs $n_{y}$ is relatively large, it may happen that for all $j \in \mathcal{S}, \operatorname{rank}\left(C_{j}\right)=$ $n$. Then, the SLS is pathwise observable with index $\nu=1$.

In short, we have seen that given a set of input-output data, the SLS model (1) cannot be uniquely determined. Since many solutions to the identification problem are possible, we may impose some contraints on the allowable solutions. A classical constraint is that of observability, which is concerned with the complexity of the model. This constraint will be particularly useful in the identification process.

Assumption 1. The switched system (1) is pathwise observable, i.e., there exists $\nu \leq \delta_{\nu}$ such that

$$
\operatorname{rank}(\Gamma(\bar{j}))=n \quad \forall \bar{j} \in \mathscr{D}_{\nu} \subset \mathcal{S}^{\nu},
$$

where $\mathscr{D}_{\nu}=\left\{\bar{\lambda}_{t, \nu} \in \mathcal{S}^{\nu} / t=0, \ldots, N-\nu\right\}$ is the set of feasible sequences of discrete states $\bar{\lambda}_{t, \nu}$ with length $\nu$.

\section{SWITCHED STATE SPACE MODEL IDENTIFICATION}

If the sequence of continuous states were completely known, the identification problem would boil down to the extraction of the parameters and the discrete state directly from the relation

$$
\left[\begin{array}{c}
x(t+1) \\
y(t)
\end{array}\right]=M_{\lambda_{t}}\left[\begin{array}{l}
x(t) \\
u(t)
\end{array}\right]
$$

where

$$
M_{\lambda_{t}}=\left[\begin{array}{c|c}
A_{\lambda_{t}} & B_{\lambda_{t}} \\
\hline C_{\lambda_{t}} & D_{\lambda_{t}}
\end{array}\right] \text { and } \lambda_{t} \in \mathcal{S} .
$$

Unfortunately, the continuous state is not measured and also needs to be computed from the input-output data. Therefore, a first step in the identification of a model for system (1) is to remove the unknown continuous state $x(t)$ from the system equations. To that end, consider the equation (6) and assume that the system order $n$ is known. Assume further that the system (1) is pathwise observable (Assumption 1). Since for any $f \geq \nu$ and for any $t, \operatorname{rank}\left(\Gamma\left(\bar{\lambda}_{t, f}\right)\right)=n$, there exists at least one weighting matrix $\Lambda\left(\bar{\lambda}_{t, f}\right) \in \mathbb{R}^{n \times f n_{y}}$ such that

$$
\operatorname{rank}\left(\Lambda\left(\bar{\lambda}_{t, f}\right) \Gamma\left(\bar{\lambda}_{t, f}\right)\right)=n .
$$

If we let $T\left(\bar{\lambda}_{t, f}\right)=\Lambda\left(\bar{\lambda}_{t, f}\right) \Gamma\left(\bar{\lambda}_{t, f}\right)$, then, by virtue of (13), $T\left(\bar{\lambda}_{t, f}\right)$ is a square nonsingular matrix. Hence, $T\left(\bar{\lambda}_{t, f}\right)$ can be used to carry out a state transformation in the model (1). By multiplying now Eq. (6) on the left by $\Lambda\left(\bar{\lambda}_{t, f}\right)$ and by setting

$$
\bar{x}(t)=T\left(\bar{\lambda}_{t, f}\right) x(t)
$$

we get

$$
\bar{x}(t)=\Lambda\left(\bar{\lambda}_{t, f}\right) y_{f}(t)-\Lambda\left(\bar{\lambda}_{t, f}\right) H\left(\bar{\lambda}_{t, f}\right) u_{f}(t) .
$$

Note that $\bar{x}(t)$ corresponds to a continuous state of the system obtained by applying a similarity transformation represented by the matrix $T\left(\lambda_{t, f}\right)$. From the state equation (15), it can be seen that for an autonomous system, $\bar{x}(t)=\Lambda\left(\bar{\lambda}_{t, f}\right) y_{f}(t)$ is a valid continuous state of the system whenever $\Lambda\left(\bar{\lambda}_{t, f}\right)$ satisfies the above rank condition. Now we can rewrite the equation (6) as

$$
\begin{aligned}
y_{f}(t) & =\Gamma\left(\bar{\lambda}_{t, f}\right) T\left(\bar{\lambda}_{t, f}\right)^{-1} T\left(\bar{\lambda}_{t, f}\right) x(t)+H\left(\bar{\lambda}_{t, f}\right) u_{f}(t) \\
& =\bar{\Gamma}\left(\bar{\lambda}_{t, f}\right) \bar{x}(t)+H\left(\bar{\lambda}_{t, f}\right) u_{f}(t) \\
& =\bar{\Gamma}\left(\bar{\lambda}_{t, f}\right)\left(\Lambda\left(\bar{\lambda}_{t, f}\right) y_{f}(t)-\Lambda\left(\bar{\lambda}_{t, f}\right) H\left(\bar{\lambda}_{t, f}\right) u_{f}(t)\right) \\
& +H\left(\bar{\lambda}_{t, f}\right) u_{f}(t) \\
= & F\left(\bar{\lambda}_{t, f}\right)\left[\begin{array}{c}
\Lambda\left(\bar{\lambda}_{t, f}\right) y_{f}(t) \\
u_{f}(t)
\end{array}\right],
\end{aligned}
$$

where

$$
F\left(\bar{\lambda}_{t, f}\right)=\left[\bar{\Gamma}\left(\bar{\lambda}_{t, f}\right)\left(I_{f n_{y}}-\bar{\Gamma}\left(\bar{\lambda}_{t, f}\right) \Lambda\left(\bar{\lambda}_{t, f}\right)\right) H\left(\bar{\lambda}_{t, f}\right)\right]
$$
is a matrix in $\mathbb{R}^{f n_{y} \times\left(n+f n_{u}\right)}$ and $\bar{\Gamma}\left(\bar{\lambda}_{t, f}\right)=\Gamma\left(\bar{\lambda}_{t, f}\right) T\left(\bar{\lambda}_{t, f}\right)^{-1}$. If we define $\pi$ as the map ${ }^{1} \pi: \mathscr{D}_{f} \longrightarrow\left\{1, \ldots,\left|\mathscr{D}_{f}\right|\right\}$ that associates to each $\bar{\lambda}_{t, f} \in \mathscr{D}_{f}$, a new discrete state $q_{t}=$ $\pi\left(\bar{\lambda}_{t, f}\right)=\pi\left(\lambda_{t} \lambda_{t+1} \cdots \lambda_{t+f-1}\right)$, in $\left\{1, \ldots,\left|\mathscr{D}_{f}\right|\right\}$, then $\pi$ is bijective. By exploiting this property, we can index the matrices $F\left(\bar{\lambda}_{t, f}\right)$ with $q_{t}$. With a slight abuse of notation, let us denote from now on, $F\left(\bar{\lambda}_{t, f}\right)$ simply as $F_{q_{t}}$.

From Eq. (16), it is possible to estimate the intermediary matrices $F_{q_{t}}$. In order to achieve this goal, we need to know

\footnotetext{
${ }^{1}$ Here, $\left|\mathscr{D}_{f}\right|$ is the cardinality of $\mathscr{D}_{f}$
} 
explicitly a set of matrices $\Lambda\left(\bar{\lambda}_{t, f}\right), t=1, \ldots, N-f+1$, that satisfy the rank conditions (13). Since $\bar{\lambda}_{t, f}$ is unknown, we cannot determine matrices $\Lambda\left(\bar{\lambda}_{t, f}\right)$ that depend on it unless we generate those matrices for any time instant $t$. However, this would have the undesirable consequence of increasing the number of possible matrices $F\left(\bar{\lambda}_{t, f}\right)$ to be estimated since then, the discrete state $q_{t}$ would take values in $\{1, \ldots, N-f+1\}$.

To avoid this problem, we consider $\Lambda\left(\bar{\lambda}_{t, f}\right)$ to be a constant matrix, i.e., independent of $\bar{\lambda}_{t, f}$ and we denote $\Lambda\left(\bar{\lambda}_{t, f}\right)=\Lambda$ $\forall t$, but with the constraint

$$
\operatorname{rank}\left(\Lambda \Gamma\left(\bar{\lambda}_{t, f}\right)\right)=n \forall t
$$

Since $\bar{\lambda}_{t, f}$ lies in a finite set, the existence of such a constant matrix $\Lambda$ is guaranteed by the following lemma.

Lemma 3. Let $\left\{A_{1}, \cdots, A_{s}\right\}$ be a finite set of matrices in $\mathbb{R}^{m \times n}$, with $m \geq n$ and $\operatorname{rank}\left(A_{i}\right)=n$ for all $i \in\{1, \ldots, s\}$. Then there exists at least one matrix $L \in \mathbb{R}^{n \times m}$ such that

$$
\operatorname{rank}\left(L A_{1}\right)=\cdots=\operatorname{rank}\left(L A_{s}\right)=n .
$$

In view of Lemma 3 , we can show that by generating $\Lambda$ at random, e.g., from a uniform distribution, the required rank conditions in (18) are satisfied almost surely. Now given $\Lambda$, Eq. (16) represents simply a switched input-output model from which $F_{q_{t}}$ can be identified.

\subsection{Estimation of the intermediary models}

In this subsection, we focus on estimating the intermediary parameter matrices $F_{q_{t}}$ from the model (16), which we rewrite as

$$
y_{f}(t)=F_{q_{t}} \varphi(t)
$$

with $\varphi(t)=\left[\left(\Lambda y_{f}(t)\right)^{\top} u_{f}(t)^{\top}\right]^{\top}$. To begin with the identification procedure, let us notice that in general, all the $\left|\mathscr{D}_{f}\right|$ submodels of (20) are not necessarily sufficiently visited by the system within the available data. Therefore, those models cannot be identified consistently by any identification algorithm. For the sake of simplicity, we discard for now such situations by assuming that the data relative to each of the $\left|\mathscr{D}_{f}\right|$ submodels are enough for identification purposes. We additionally make the following assumption that assures that given a couple $\left(\varphi(t), y_{f}(t)\right)$, there is only one submodel of (20) that fits it.

Assumption 2. For any time index $t$, and for any $(i, j) \in \mathcal{S}^{2}$ with $i \neq j,\left(F_{i}-F_{j}\right) \varphi(t) \neq 0$.

Let now $s_{o}=\left|\mathscr{D}_{f}\right|$ be the number of discrete states in (20). To estimate the matrices $F_{j}$, we propose to use the following algorithm that alternates between assigning data to submodel and updating the parameters via recursive least squares:

(1) Initialize the parameter matrices $\hat{F}_{j}, j=1, \ldots, s_{o}$ at random. Initialize also, for all $j$, the correlation matrices $L_{j}=\mathbb{E}\left\{\varphi(t) \varphi(t)^{\top} \mid \lambda_{t}=j\right\}^{-1}, j=1, \ldots, s_{o}$. Denote respectively by $\hat{F}_{j}(0)$ and $L_{j}(0)=\alpha I$ these prior values.

(2) For any pair $\left(\varphi(t), y_{f}(t)\right)$ of observations,

- Estimate the discrete state as

$$
\hat{q}_{t}=\underset{j=1, \ldots, s_{o}}{\arg \min } \frac{1}{\delta_{j}(t-1)}\left\|y_{f}(t)-\hat{F}_{j}(t-1) \varphi(t)\right\|_{2},
$$

with $\delta_{j}(t-1)=\sqrt{f n_{y}+\operatorname{trace}\left(\hat{F}_{j}(t-1)^{\top} \hat{F}_{j}(t-1)\right)}$. In fact, the quantity $\delta_{j}(t-1)$ is the Frobenius matrix norm of $\left[I_{f n_{y}} \hat{F}_{j}(t-1)\right]$ and so, the division by $\delta_{j}(t-1)$ in the criterion (21) corresponds just to a normalization.

- Update the parameter matrices $\hat{F}_{\hat{q}_{t}}(t-1)$ using, e.g., Recursive Least Squares (RLS) [18] :

$$
\begin{aligned}
& \left\{\hat{F}_{\hat{q}_{t}}(t), L_{\hat{q}_{t}}(t)\right\} \\
& \quad=\operatorname{RLS}\left\{\hat{F}_{\hat{q}_{t}}(t-1), L_{\hat{q}_{t}}(t-1),\left(\varphi(t), y_{f}(t)\right)\right\},
\end{aligned}
$$

- The other submodels indexed by $j \neq \hat{q}_{t}$ remain unchanged.

(3) Go to step (2) until all the data are treated.

If we denote by $N$ the number of available input-output data $(u(t), y(t))$, then $\hat{F}_{j}\left(N_{o}\right), j=1, \ldots, s, N_{o}=N-f+1$, are the estimates obtained after the algorithm processes all the data (Steps 2 and 3). Based on these final estimates we can reconstruct the discrete state as

$$
\hat{q}_{t}=\underset{j=1, \ldots, s_{o}}{\arg \min } \frac{1}{\delta_{j}\left(N_{o}\right)}\left\|y_{f}(t)-\hat{F}_{j}\left(N_{o}\right) \varphi(t)\right\|_{2},
$$

for all $t=1, \ldots, N_{o}$. Given $\hat{q}_{t}$, one can decide depending on the value of the performance criterion

$$
\mathscr{C}\left(\left\{\hat{F}_{j}\right\}_{j=1}^{s_{o}}\right)=\sum_{t=1}^{N_{o}} \frac{1}{\delta_{\hat{q}_{t}}\left(N_{o}\right)}\left\|y_{f}(t)-\hat{F}_{\hat{q}_{t}}\left(N_{o}\right) \varphi(t)\right\|_{2}
$$

whether it is necessary to re-run the algorithm. Note that the initialization is a crucial step for the convergence of this algorithm. Since the algorithm is randomly initialized, the results obtained may be non deterministic so that several trials may be necessary to achieve good estimates. With a large amount of data, our algorithm seems to converge on an average of one trial out of three in practice.

\subsection{Extraction of the system matrices}

Given the matrices $F_{q_{t}}$ of the model (16), we are now interested in extracting a state space realization for the SLS (1). To that end, let us partition the matrix $F_{q_{t}}$ as $F_{q_{t}}=\left[\begin{array}{ll}F_{q_{t}}^{y} & F_{q_{t}}^{u}\end{array}\right]$ such that $F_{q_{t}}^{y}$ and $F_{q_{t}}^{u}$ contain respectively $n$ and $f n_{u}$ columns. Then, by using the definition of $F_{q_{t}}$ in (16), one can obtain the observability matrix $\bar{\Gamma}\left(\bar{\lambda}_{t, f}\right)$ directly as $\bar{\Gamma}\left(\bar{\lambda}_{t, f}\right)=F_{q_{t}}^{y}$.

Computing $H\left(\bar{\lambda}_{t, f}\right)$ requires, on the other hand, more involved calculations (See Proposition 4 below). This is due to the fact that the matrix $I_{f n_{y}}-\bar{\Gamma}\left(\bar{\lambda}_{t, f}\right) \Lambda$ is singular and so, $H\left(\bar{\lambda}_{t, f}\right)$ cannot be obtained directly from $F_{q_{t}}^{u}$. In fact, for any matrix $\Lambda$ satisfying (18), it can be verified that

$$
\operatorname{rank}\left(I_{f n_{y}}-\bar{\Gamma}\left(\bar{\lambda}_{t, f}\right) \Lambda\right)=f n_{y}-n .
$$

In order to extract the system matrices, we recall that the state transformation (14) produces a shift in both the matrices and the number of discrete states of model (1). More precisely, the initial switched model $\left\{A_{\lambda_{t}}, B_{\lambda_{t}}, C_{\lambda_{t}}, D_{\lambda_{t}}\right\}, \lambda_{t} \in \mathcal{S}$, is transformed (without any modification of the input-output behavior) into another switched model $\left\{\bar{A}_{p_{t}}, \bar{B}_{p_{t}}, \bar{C}_{p_{t}}, \bar{D}_{p_{t}}\right\}$, $p_{t} \in\left\{1, \ldots,\left|\mathscr{D}_{f+1}\right|\right\}$ such that

$$
\left[\begin{array}{c|c}
\bar{A}_{p_{t}} & \bar{B}_{p_{t}} \\
\hline \bar{C}_{p_{t}} & \bar{D}_{p_{t}}
\end{array}\right]=\left[\begin{array}{c|c}
T\left(\bar{\lambda}_{t+1, f}\right) A_{\lambda_{t}} T\left(\bar{\lambda}_{t, f}\right)^{-1} & T\left(\bar{\lambda}_{t+1, f}\right) B_{\lambda_{t}} \\
\hline C_{\lambda_{t}} T\left(\bar{\lambda}_{t, f}\right)^{-1} & D_{\lambda_{t}}
\end{array}\right]
$$

with $T\left(\bar{\lambda}_{t, f}\right)=\Lambda \Gamma\left(\bar{\lambda}_{t, f}\right)$ and $p_{t} \in\left\{1, \ldots,\left|\mathscr{D}_{f+1}\right|\right\}$. Here, the discrete state $p_{t}$ is defined based on the bijective correspondence between the finite set of discrete state sequences involved 
in $T\left(\bar{\lambda}_{t+1, f}\right) A_{\lambda_{t}} T\left(\bar{\lambda}_{t, f}\right)^{-1},\left\{\bar{\lambda}_{t, f+1}: t=1, \ldots, N-f\right\}$, and the set $\left\{1, \ldots,\left|\mathscr{D}_{f+1}\right|\right\}$.

Remark 2. In (25), the discrete state $p_{t}$ takes values in the finite set $\left\{1, \ldots,\left|\mathscr{D}_{f+1}\right|\right\}$, where $\mathscr{D}_{f+1} \subset \mathcal{S}^{f}$ is the set of feasible paths of length $f+1$, while $q_{t}$ from (20) takes values in $\left\{1, \ldots,\left|\mathscr{D}_{f}\right|\right\}$. In fact, we can see from Eq. (25) that $\bar{C}_{p_{t}}$ and $\bar{D}_{p_{t}}$ can be indexed with $q_{t}=\pi\left(\bar{\lambda}_{t, f}\right)$. The matrix $\bar{D}_{p_{t}}$ can even be indexed by the discrete state $\lambda_{t} \in \mathcal{S}$, because it takes at most $s$ distinct values $\left(\bar{D}_{p_{t}}=D_{\lambda_{t}}\right)$. But the matrices $\bar{A}_{p_{t}}$ and $\bar{B}_{p_{t}}$, which depend on both $q_{t}$ and $q_{t+1}$, can take $\left|\mathscr{D}_{f+1}\right| \geq\left|\mathscr{D}_{f}\right|$ different values.

By setting $f=\nu+1$, the matrices in (25) can be computed thanks to Proposition 4 below. In order to state this proposition, we need to introduce the notation

$$
\mathcal{K}_{q_{t}}=I_{f n_{y}}-\bar{\Gamma}\left(\bar{\lambda}_{t, f}\right) \Lambda=I_{f n_{y}}-F_{q_{t}}^{y} \Lambda,
$$

and consider the following partitions

$$
\begin{aligned}
& F_{q_{t}}^{y}=\left[\begin{array}{lll}
\left(F_{q_{t}}^{y, 1}\right)^{\top} \cdots & \left(F_{q_{t}}^{y, f}\right)^{\top}
\end{array}\right]^{\top} \in \mathbb{R}^{f n_{y} \times n}, \\
& F_{q_{t}}^{y, \alpha: \beta}=\left[\begin{array}{lll}
\left(F_{q_{t}}^{y, \alpha}\right)^{\top} \cdots & \left(F_{q_{t}}^{y, \beta}\right)^{\top}
\end{array}\right]^{\top} \in \mathbb{R}^{(\beta-\alpha+1) n_{y} \times n}, \\
& \mathcal{K}_{q_{t}}=\left[\begin{array}{ll}
\mathcal{K}_{q_{t}}^{1} \cdots & \mathcal{K}_{q_{t}}^{f}
\end{array}\right] \in \mathbb{R}^{f n_{y} \times f n_{y}}, \\
& F_{q_{t}}^{u}=\left[\begin{array}{lll}
F_{q_{t}}^{u, 1} \cdots & F_{q_{t}}^{u, f}
\end{array}\right] \in \mathbb{R}^{f n_{y} \times f n_{u}},
\end{aligned}
$$

where $F_{q_{t}}^{y, k} \in \mathbb{R}^{n_{y} \times n}, \mathcal{K}_{q_{t}}^{k} \in \mathbb{R}^{f n_{y} \times n_{y}}, F_{q_{t}}^{u, k} \in \mathbb{R}^{f n_{y} \times n_{u}}$, $k=1, \ldots, f$, and $1 \leq \alpha \leq \beta \leq f$.

Proposition 4. Given the matrices $F_{j}, j=1, \ldots, s$, and the discrete state $q_{t}$ of the model (20), the following holds for all $t \geq f-1$

$$
\left\{\begin{array}{l}
\bar{A}_{p_{t}}=\left(F_{q_{t+1}}^{y, 1: f-1}\right)^{\dagger} F_{q_{t}}^{y, 2: f} \\
\bar{C}_{p_{t}}=F_{q_{t}}^{y, 1}, \\
{\left[\begin{array}{c}
\bar{D}_{p_{t}} \\
\bar{B}_{p_{t}}
\end{array}\right]=\left[\mathscr{K}\left(\bar{q}_{t-f+1, f}\right) \mathcal{R}_{q_{t+1}}\right]^{\dagger}\left[\begin{array}{c}
F_{q_{t-f+1}^{u}, f}^{u} \\
F_{q_{t-f+2}}^{u, f-1} \\
\vdots \\
F_{q_{t}}^{u, 1}
\end{array}\right],}
\end{array}\right.
$$

where

and

$$
\mathcal{R}_{q_{t+1}}=\left[\begin{array}{cc}
I_{n_{y}} & \mathrm{O}_{n_{y}, n} \\
\mathrm{O}_{(f-1) n_{y}, n_{y}} & F_{q_{t+1}, 1: f-1}^{y, 1}
\end{array}\right]
$$

$\mathscr{K}\left(\bar{q}_{t-f+1, f}\right)=\left[\begin{array}{ccccc}\mathcal{K}_{q_{t-f+1}}^{f} & \mathrm{O}_{f n_{y}, n_{y}} & \cdots & \mathrm{O}_{f n_{y}, n_{y}} & \mathrm{O}_{f n_{y}, n_{y}} \\ \mathcal{K}_{q_{t-f+2}}^{f-1} & \mathcal{K}_{q_{t-f+2}}^{f} & \cdots & \mathrm{O}_{f n_{y}, n_{y}} & \mathrm{O}_{f n_{y}, n_{y}} \\ \vdots & \vdots & \ddots & \vdots & \vdots \\ \mathcal{K}_{q_{t-1}}^{2} & \mathcal{K}_{q_{t-1}}^{3} & \cdots & \mathcal{K}_{q_{t-1}}^{f} & \mathrm{O}_{f n_{y}, n_{y}} \\ \mathcal{K}_{q_{t}}^{1} & \mathcal{K}_{q_{t}}^{2} & \cdots & \mathcal{K}_{q_{t}}^{f-1} & \mathcal{K}_{q_{t}}^{f}\end{array}\right]$ where $\bar{q}_{t-f+1, f}=q_{t-f+1} \cdots q_{t}$ is a sequence of discrete states and $\mathrm{O}_{f n_{y}, n_{y}}$ is a matrix in $\mathbb{R}^{f n_{y} \times n_{y}}$ whose entries are zero.

Remark 3. Eq. (30) holds regardless of the switching mechanism, i.e., the switches can be very arbitrary. Stability of the global SLS is not explicitly required, but may be important for the numerical conditioning of the data matrices. Since the formulae (30) are established under general assumptions, they also apply to the particular case where the switching times are separated by a minimum duration.

In order to apply Proposition 4, it is necessary that the matrices $F_{q_{t}}$ be well estimated and that the discrete state be recovered exactly, at least on a certain time horizon where all the $\left|\mathscr{D}_{f+1}\right|$ submodels show up. If this is the case, then the estimated submodel matrices $\left\{\bar{A}_{j}, \bar{B}_{j}, \bar{C}_{j}, \bar{D}_{j}\right\}, j=1, \ldots,\left|\mathscr{D}_{f+1}\right|$ are naturally consistent in the sense that the state coordinates bases of all the submodels are such that the state transformations $T\left(\bar{\lambda}_{t, f}\right)$ mutually compensate (see Eq. (25)). In this way, the input-output map (2) does not change if we replace $x(0)$ with $\bar{x}(0)$ and $\left(A_{\lambda_{t}}, B_{\lambda_{t}}, C_{\lambda_{t}}, D_{\lambda_{t}}\right)$ with $\left(\bar{A}_{p_{t}}, \bar{B}_{p_{t}}, \bar{C}_{p_{t}}, \bar{D}_{p_{t}}\right)$.

Now, given the state space model $\left\{\bar{A}_{p_{t}}, \bar{B}_{p_{t}}, \bar{C}_{p_{t}}, \bar{D}_{p_{t}}\right\}, p_{t} \in$ $\left\{1, \ldots,\left|\mathscr{D}_{f+1}\right|\right\}$ in (30), how can we find a realization of the form $\left\{A_{\lambda_{t}}, B_{\lambda_{t}}, C_{\lambda_{t}}, D_{\lambda_{t}}\right\}, \lambda_{t} \in \mathcal{S}$, for system (1)? Obviously, the response to this question would immediately be affirmative if the transformation matrices $T\left(\bar{\lambda}_{t, f}\right)$ were all known or equal. Unfortunately this is not the case in general. However there are some particular cases where there exists a direct correspondence between the two realizations.

Example 1. Consider a MISO system of the form (1) with parameters

$$
C_{\lambda_{t}}=\left[\begin{array}{llll}
1 & 0 & \cdots & 0
\end{array}\right] \text { and } A_{\lambda_{t}}=\left[\begin{array}{cc}
\mathrm{O}_{n-1,1} & I_{n-1} \\
-a_{\lambda_{t}}^{0} & -a_{\lambda_{t}}^{\top}(2: n)
\end{array}\right] \text {, }
$$

where $a_{\lambda_{t}}^{\top}=\left[\begin{array}{lll}a_{\lambda_{t}}^{0} \cdots & a_{\lambda_{t}}^{n-1}\end{array}\right]$, for all $\lambda_{t} \in \mathcal{S}$, and the matrices $B_{\lambda_{t}}$ and $C_{\lambda_{t}}$ are arbitrary. Then for any $\bar{\lambda}_{t, n}$, we have $\Gamma\left(\bar{\lambda}_{t, n}\right)=I_{n}$ regardless of the switching mechanism. It follows that if we let $f=n+1$, we can take $\Lambda=$ $\left[\begin{array}{ll}I_{n} & 0_{n, 1}\end{array}\right]$ so that $T\left(\bar{\lambda}_{t, f}\right)=\Lambda \Gamma\left(\bar{\lambda}_{t, f}\right)=I_{n}$ for all $\bar{\lambda}_{t, n}$. As a consequence, Eq. (25) implies that $\left(\bar{A}_{p_{t}}, \bar{B}_{p_{t}}, \bar{C}_{p_{t}}, \bar{D}_{p_{t}}\right)=$ $\left(A_{\lambda_{t}}, B_{\lambda_{t}}, C_{\lambda_{t}}, D_{\lambda_{t}}\right)$.

More generally, reducing the realization (30) (with $\left|\mathscr{D}_{f+1}\right|$ submodels) to a realization with a minimal number of submodels (i.e. $s$ submodels) may require the application of some more involved techniques. This model reduction step is out of the scope of this paper and will be considered in future work.

\subsection{Particular case with dwell time}

Our method applies also to the particular case where there is a certain minimum duration between consecutive switches. In this case, the algorithm of Subsection 3.1 can be made more efficient by using for example a sliding window to carry out the estimation and clustering tasks. For example, we can start the algorithm with one submodel, while watching the variance of the estimated parameter matrix $\hat{F}_{1}$. Then, after convergence, a switch can be detected, because it will cause the variance to jump. Whenever a switch is detected, we can increment the number of submodels and continue the procedure by alternating between updating the two submodels according to a decision criterion such as (21).

Another approach may be as follows. If we assume that the dwell time is large enough, then the switches occur so rarely that (mixed) discrete states of the form $q_{t}=\pi\left(\lambda_{t} \cdots \lambda_{t+f-1}\right)$ where the states $\lambda_{t}, \ldots, \lambda_{t+f-1}$ are not all equal, are not sufficiently excited. Therefore, as done in [11], we can neglect those discrete states so that the model (20) can be regarded as having the same number of submodels as (1). By doing so, it is necessary to set up some appropriate thresholds for robustly removing the small number of data (now treated as outliers) that are generated by mixed submodels.

\section{NUMERICAL EXAMPLE}

To illustrate our method, we consider an SLS consisting of $s=2$ submodels of order $n=2$ defined by 


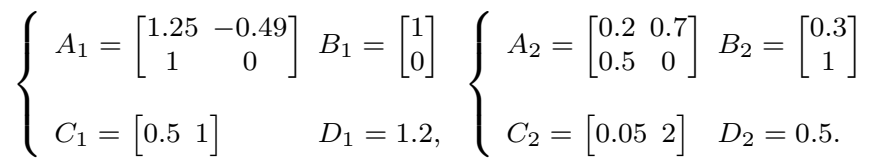

We take the weighting matrix $\Lambda$ to be $\Lambda=\left[\begin{array}{lll}1 & 0 & 1 \\ 0 & 1 & 1\end{array}\right]$. The exciting input signal is chosen to be a zero-mean white noise with unit variance. We set the tuning parameter $f$ to be equal to 3 . Then the model (20) contains at most $s^{f}=8$ submodels. The switching sequence is generated at random in such a way that all the 8 submodels are sufficiently excited in a collection of input-output data of size $N=100,000$. A certain amount of white noise is finally added to the output so that we have an SNR equal to $30 \mathrm{~dB}$. We depict in Figure 1, the evolution of

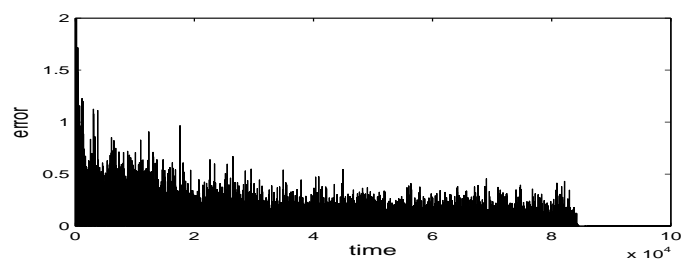

(a) Without noise

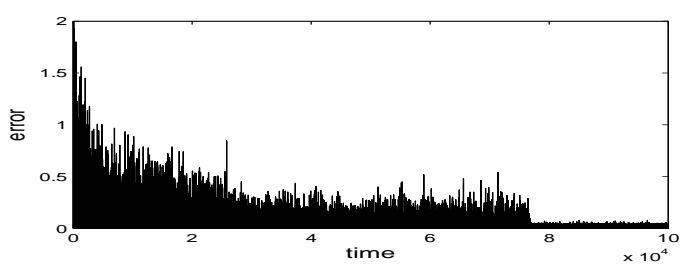

(b) With noise

Figure 1. Convergence of the algorithm of Subsection 3.1.

the minimum of the decision criterion (21) in both the noisefree and noisy cases. This prediction error does not decrease monotonically because the 8 identification algorithms that work in parallel do not converge at the same time. One can notice that when convergence occurs (after 80,000 samples), the error reaches a minimum value. This minimum value is much smaller (almost zero) in the noise-free case. By extracting the state space model in (25), we obtain 16 different values for matrices $A_{p_{t}}$ and $B_{p_{t}}, 8$ and 2 different values for respectively $C_{p_{t}}$ and $D_{p_{t}}$.

\section{CONCLUDING REMARKS}

We have proposed a method for handling the challenging problem of estimating a switched linear state space model from data. From our discussion, it appears that this problem involves a number of subsidiary difficulties that are essentially related to the fact that the continuous state is unknown. By eliminating, under a certain observability condition, the continuous state from the system equations, we get a switched input-output model that involves an increased number of discrete states. The estimation of this latter input-output model allows us to extract a realization of the data-generating switched state space model. In comparison with existing techniques, our method applies to the general case where the system may switch arbitrarily fast and provides a very natural solution to the problem of matching the state coordinates bases of the estimated submodels.

\section{REFERENCES}

[1] G. Ferrari-Trecate, M. Muselli, D. Liberati, and M. Morari, "A clustering technique for the identification of piecewise affine systems," Automatica, vol. 39, pp. 205-217, 2003.

[2] J. Roll, A. Bemporad, and L. Ljung, "Identification of piecewise affine systems via mixed-integer programming," Automatica, vol. 40, pp. 37-50, 2004.

[3] A. Bemporad, A. Garulli, S. Paoletti, and A. Vicino, "A bounded-error approach to piecewise affine system identification," IEEE Transactions on Automatic Control, vol. 50, pp. 1567-1580, 2005.

[4] A. L. Juloski, S. Weiland, and W. Heemels, "A bayesian approach to identification of hybrid systems," IEEE Transactions on Automatic Control, vol. 50, pp. 15201533, 2005.

[5] R. Vidal, S. Soatto, Y. Ma, and S. Sastry, "An algebraic geometric approach to the identification of a class of linear hybrid systems," in Conference on Decision and Control, Maui, Hawaii, USA, 2003.

[6] Y. Ma and R. Vidal, "Identification of deterministic switched arx systems via identification of algebraic varieties," in Hybrid systems computation and control, Zurich, Switzerland, 2005.

[7] L. Bako and R. Vidal, "Algebraic identification of switched MIMO ARX models," in Hybrid Systems: Control and Computation, St Louis MO, USA, 2008.

[8] S. Paoletti, A. Juloski, G. Ferrari-Trecate, and R. Vidal., "Identification of hybrid systems: A tutorial.," European Journal of Control, vol. 13, pp. 242-260, 2007.

[9] L. Bako, G. Mercère, and S. Lecoeuche, "Online structured subspace identification with application to switched linear systems," International Journal of Control (To appear), 2009.

[10] J. Borges, V. Verdult, M. Verhaegen, and M. A. Botto, "A switching detection method based on projected subspace classification," in Conference on Decision and ControlEuropean Control Conference, Seville, Spain, 2005.

[11] K. Huang, A. Wagner, and Y. Ma, "Identification of hybrid linear time-invariant systems via subspace embedding and segmentation," in Conference on Decision and Control, Atlantis, Paradise Island, Bahamas, 2004.

[12] V. Verdult and M. Verhaegen, "Subspace identification of piecewise linear systems," in Conference on Decision and Control, Atlantis, Paradise Island, Bahamas, 2004.

[13] M. Petreczky, Realization theory of hybrid systems. PhD thesis, Vrije Universiteit, Amsterdam, 2006.

[14] R. Vidal, A. Chiuso, and S. Soatto., "Observability and identifiability of jump linear systems," in Conference on Decision and Control, Las Vegas NV, 2002, 2002.

[15] S. Paoletti, J. Roll, A. Garulli, and A. Vicino, "Inputoutput realization of piecewise affine state space models," in Conference on Decision and Control, New Orleans LA, USA, 2007.

[16] S. Weiland, A. L. Juloski, and B. Vet, "On the equivalence of switched affine models and switched ARX models," in Conference on Decision and Control, San Diego CA, USA, 2006.

[17] M. Babaali and M. Egerstedt, "Observability for switched linear systems," in Hybrid Systems: Computation and Control, Philadelphia PA, USA, 2004.

[18] L. Ljung and T. Söderström, Theory and Practice of Recursive Identification. MIT Press, Cambridge, 1983. 